\title{
Spatial Algorithms at Scale with Spatialpandas
}

\author{
Dharhas Pothina \\ Kim Pevey \\ Adam Lewis
}

\section{Motivation}

- Large Geospatial Dataset (1 trillion rows of point data and growing)

- Data is stored in flat files

- Need to efficiently run spatially localized queries

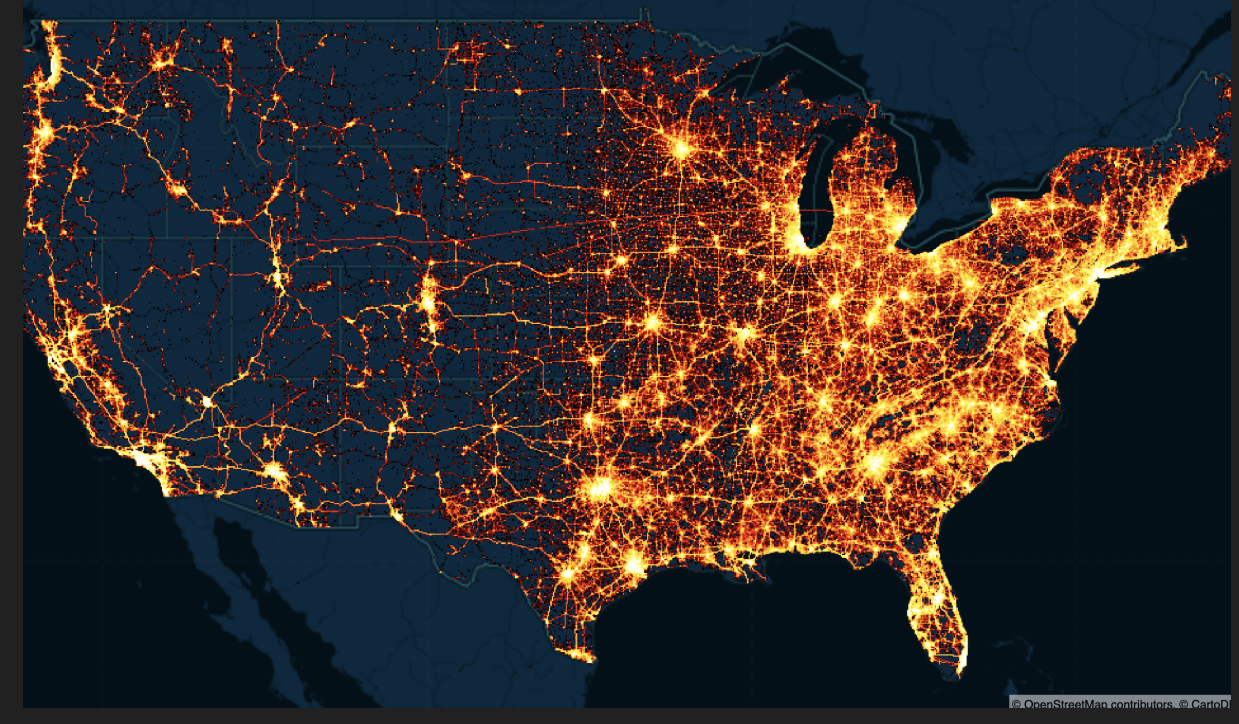




\section{Surrogate Dataset}

- All GPS data from OpenStreetMap from June $2012^{1}$

○ 2.9 billion latitude/longitude point pairs only

- 73 GB Uncompressed CSV File

- Contributed by Thousands of Users

- Contiguous US subset will be used in this presentation

- 114 million rows

- 3.2 GB Uncompressed CSV File

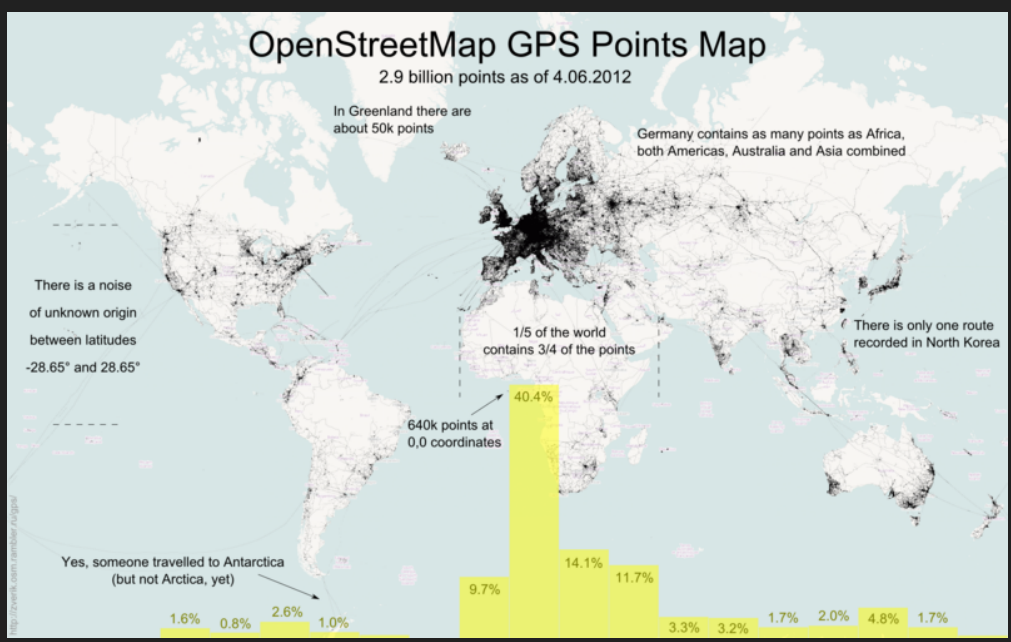

1. Freely available for download from https://planet.openstreetmap.org/gps/simple-gps-points-120604.csv.XZ

\section{Problems}

\section{Kernel Restarting}

The kernel for visualizations/pandas_crash.ipynb appears to have died. It will restart automatically.

df $=$ pd.read_csv(' . ./data/simple-gps-points-120604.csv')

1. The dataset is too big to fit in memory

2. Searching through the entire dataset is slow

a. A naive approach touches every data point during the search

b. Reading from disk is slow

c. Finding if all points are within a polygon is computationally expensive 


\section{Solution}

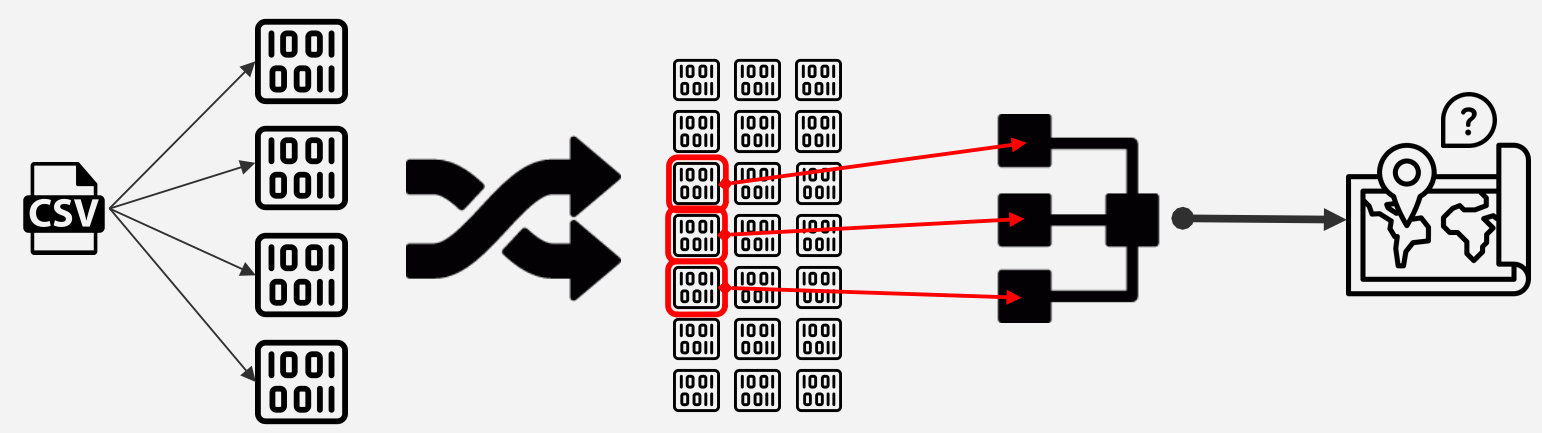

1. Use multiple smaller binary files or partitions rather than a single csv file

2. Sort the dataset so points that are spatially near are stored in the same file

3. Build a global index so only relevant files loaded based on the local region being queried

4. Conduct queries in parallel

\section{Spatial Sort}

- Need to map 2D space (lat,long) --> 1D Space

- The 1D variable must have the property that values close to another are also spatially close to each other.

- Options: Geohash, Hilbert Space Filling Curve, Google S2, Uber H3,

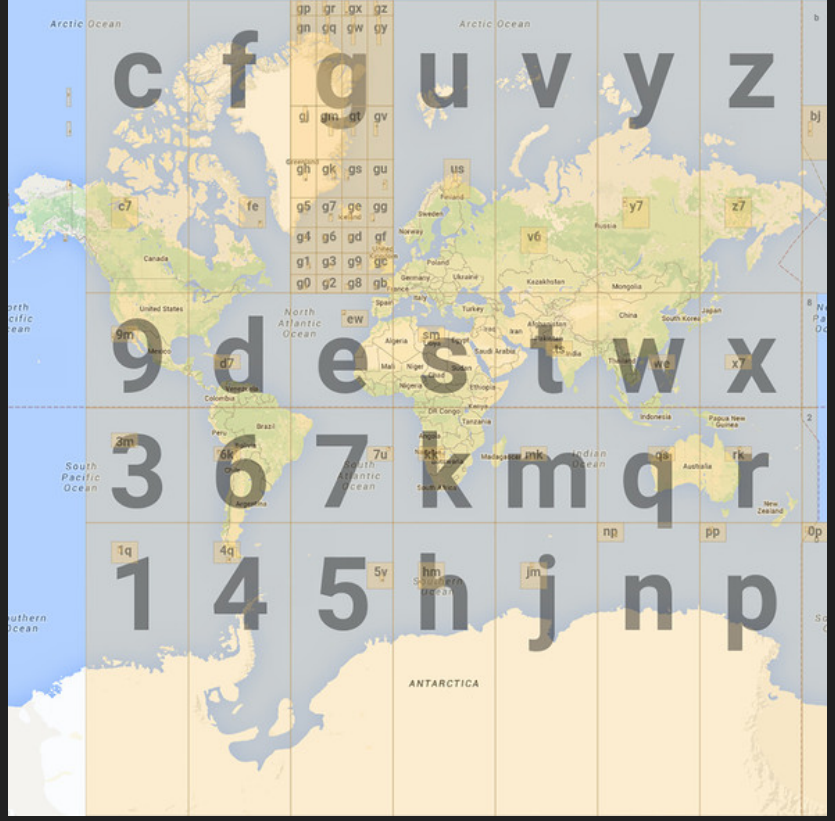

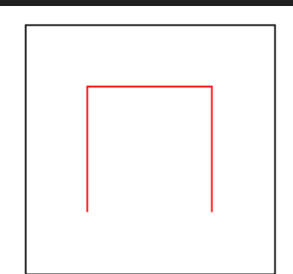

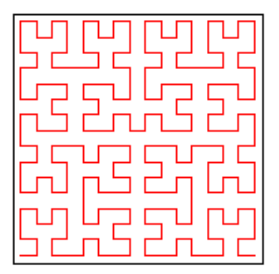

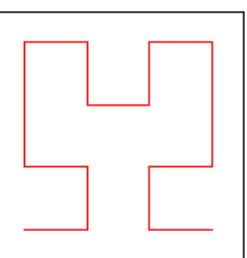
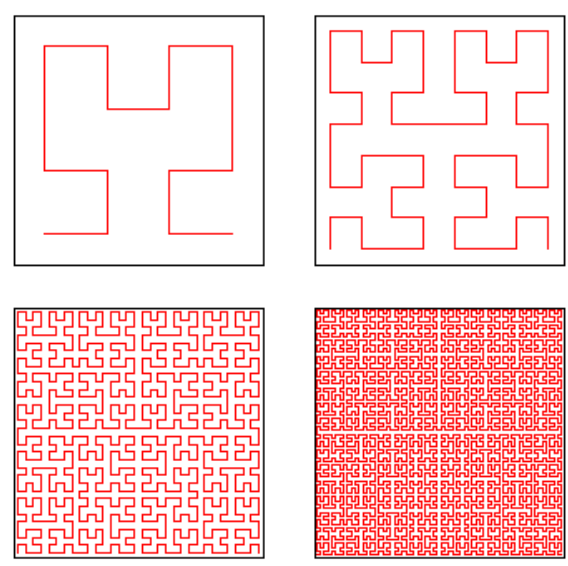


\section{Geohash}

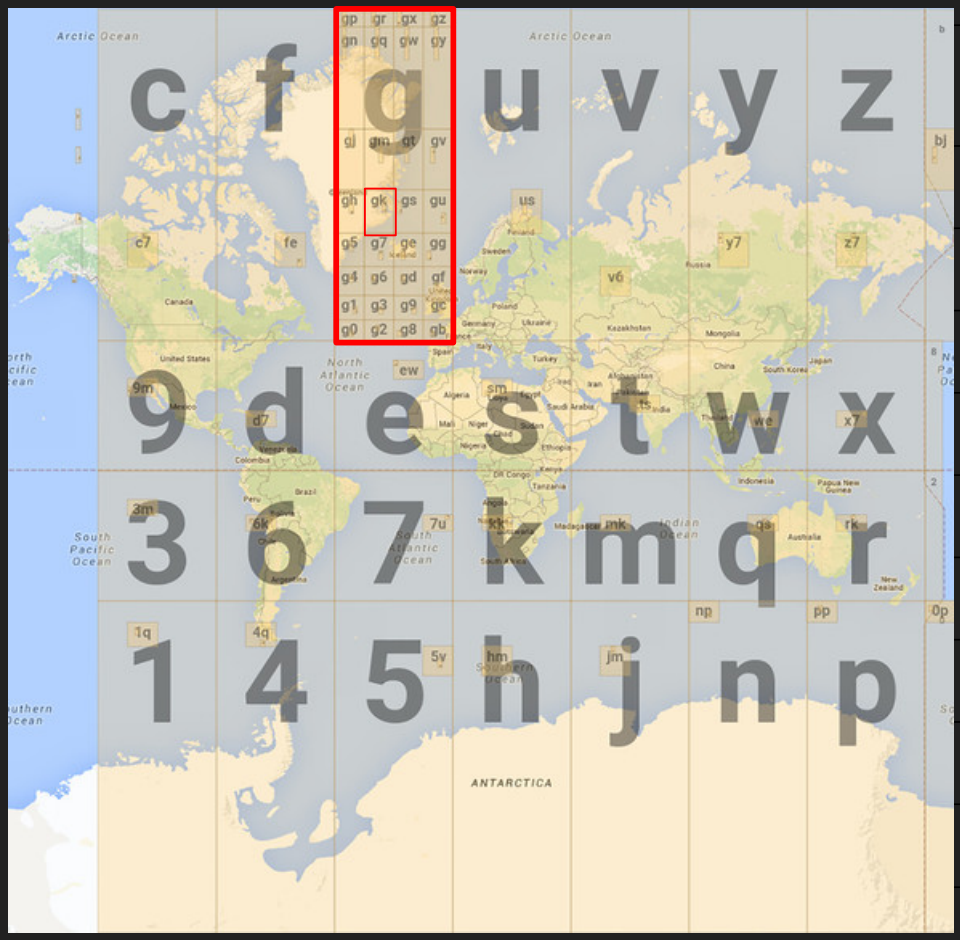

\section{\# Geohash Example}

Cell width

Cell Height

Character

Geohash

\begin{tabular}{|l|l|l|l|l|}
\hline 1 & $\mathrm{~g}$ & $\leq 5,000 \mathrm{~km}$ & $\mathrm{x}$ & $5,000 \mathrm{~km}$ \\
\hline 2 & $\mathrm{gk}$ & $\leq 1,250 \mathrm{~km}$ & $\mathrm{x}$ & $625 \mathrm{~km}$ \\
\hline 3 & $\mathrm{gk6}$ & $\leq 156 \mathrm{~km}$ & $\mathrm{x}$ & $156 \mathrm{~km}$ \\
\hline 4 & gk6k & $\leq 39.1 \mathrm{~km}$ & $\mathrm{x}$ & $19.5 \mathrm{~km}$ \\
\hline 5 & gk6kp & $\leq 4.89 \mathrm{~km}$ & $\mathrm{x}$ & $4.89 \mathrm{~km}$ \\
\hline 6 & gk6kpt & $\leq 1.22 \mathrm{~km}$ & $\mathrm{x}$ & $0.61 \mathrm{~km}$ \\
\hline 7 & gk6kptw & $\leq 153 \mathrm{~m}$ & $\mathrm{x}$ & $153 \mathrm{~m}$ \\
\hline 8 & gk6ktpw9 & $\leq 38.2 \mathrm{~m}$ & $\mathrm{x}$ & $19.1 \mathrm{~m}$ \\
\hline 9 & gk6ktpw9h & $\leq 4.77 \mathrm{~m}$ & $\mathrm{x}$ & $4.77 \mathrm{~m}$ \\
\hline
\end{tabular}

https://en.wikipedia.org/wiki/Geohash

\section{Hilbert Curve}

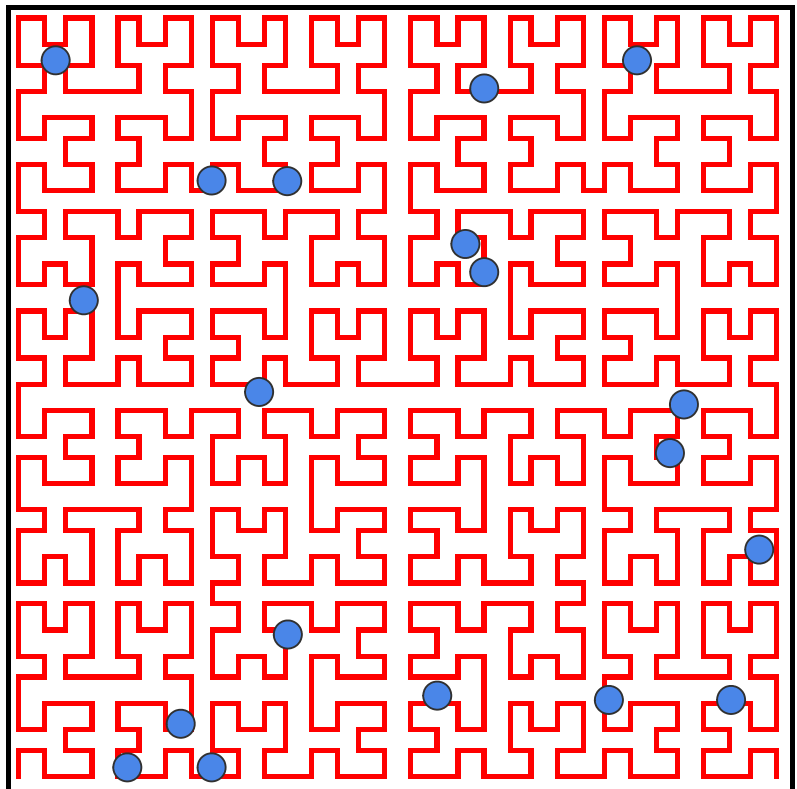




\section{Solution (Tech Stack)}

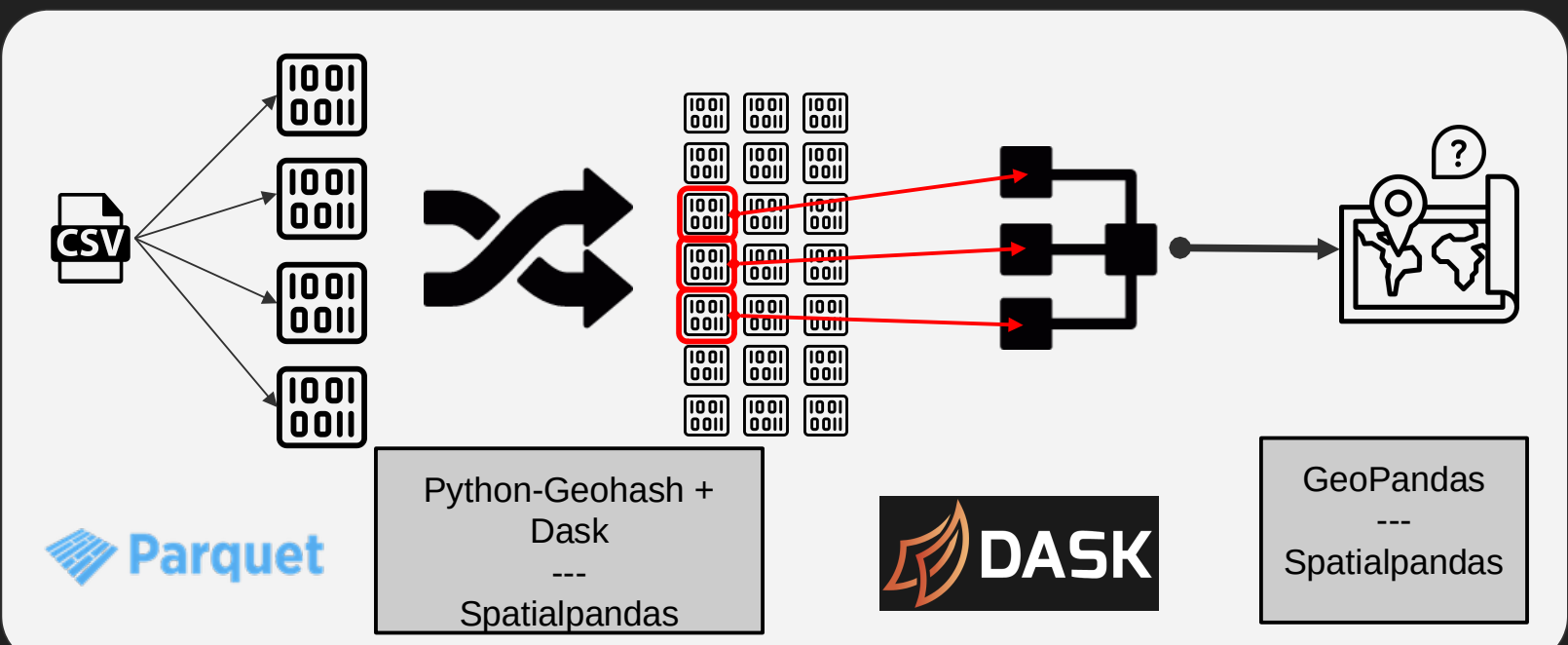

1. Use multiple smaller binary files or partitions rather than a single csv file

2. Sort the dataset so points that are spatially near are stored in the same file

3. Build a global index so only relevant files loaded based on the local region being queried

4. Conduct queries in parallel

\section{Benchmark:}

Machine Specifications

- Processor: AMD Ryzen Threadripper 2970WX 24-Core Processor

- RAM: 64 GB

- Shared System

- For this comparison Dask Workers were limited to

- 4 Workers
- 2 Threads per Worker
- 3 GB RAM per Worker

- Final computation brings the filtered data into the main process

\section{Dataset}

- OpenStreetMaps Unsorted Contiguous US dataset in parquet file format.

Preprocess \& Queries

- Preprocess the Data (if necessary)

- Sort the Data

- Select points from dataset that are within 1, 10, 100, 1000, 10000 random zip code polygons distributed around the US

Publicly available: https://www2.census.gov/geo/tiger/TIGER2019/ZCTA5/tI_2019_us_zcta510.zip 


\section{Unsorted (Naive Approach)}

- Preprocess data if necessary:

- N/A

- Sort data if necessary

- N/A

- Spatially Index into Data

- Apply Geopandas's sjoin* method to each partition of GPS data in Dask

* spatial joining finds points in the dataset that are inside the zip code polygons

\section{Unsorted Case}

\begin{tabular}{|c|c|c|c|c|c|c|}
\hline \# Polygons & \# Points & $\begin{array}{c}\text { Geohash } \\
\text { Time (s) }\end{array}$ & $\begin{array}{l}\text { Sort Time } \\
\text { (s) }\end{array}$ & $\begin{array}{l}\text { Query Time } \\
\text { (min) }\end{array}$ & $\begin{array}{l}\text { \# Result } \\
\text { Points }\end{array}$ & $\begin{array}{l}\text { Total Time } \\
\text { (min) }\end{array}$ \\
\hline 1 & 113944489 & \multirow{3}{*}{0} & \multirow{3}{*}{0} & 41 & 1031 & 41 \\
\hline 10 & 113944489 & & & 41 & 6551 & 41 \\
\hline 100 & 113944489 & & & 47 & 203284 & 47 \\
\hline 1000 & \multicolumn{6}{|c|}{ Not enough memory } \\
\hline 10000 & & & \multicolumn{2}{|c|}{ Not enough memory } & & \\
\hline
\end{tabular}




\section{Sorted Geohash}

- Preprocess data if necessary:

- Compute Geohash

- 4 character geohash used

- Tradeoffs

- More precise geohash, increased geohash calculation time, reduced sjoin time

- Sort data

- Sort Geohash Lexicographically using Dask

- Spatially Index into Data

- Calculate all Geohashes for Each Polygon

- Index into data by polygon geohashes

- Only need to read the partitions with data in those geohashes

- Apply GeoPandas's sjoin method to each resulting partition of points

\section{Sorted Geohash No Sjoin (Less Accurate)}

- Preprocess data if necessary:

- Compute Geohash

- 4 character geohash used

- Tradeoffs

- More precise geohash, increased geohash calculation time, reduced sjoin time

- Sort data

- Sort Geohash Lexicographically using Dask

- Spatially Index into Data

- Calculate all Geohashes for Each Polygon

- Index into data by polygon geohashes

- Only need to read the partitions with data in those geohashes

- Apply GeoPandas's sjoin method to each resulting partition of points 


\section{Comparison of Resulting Points}

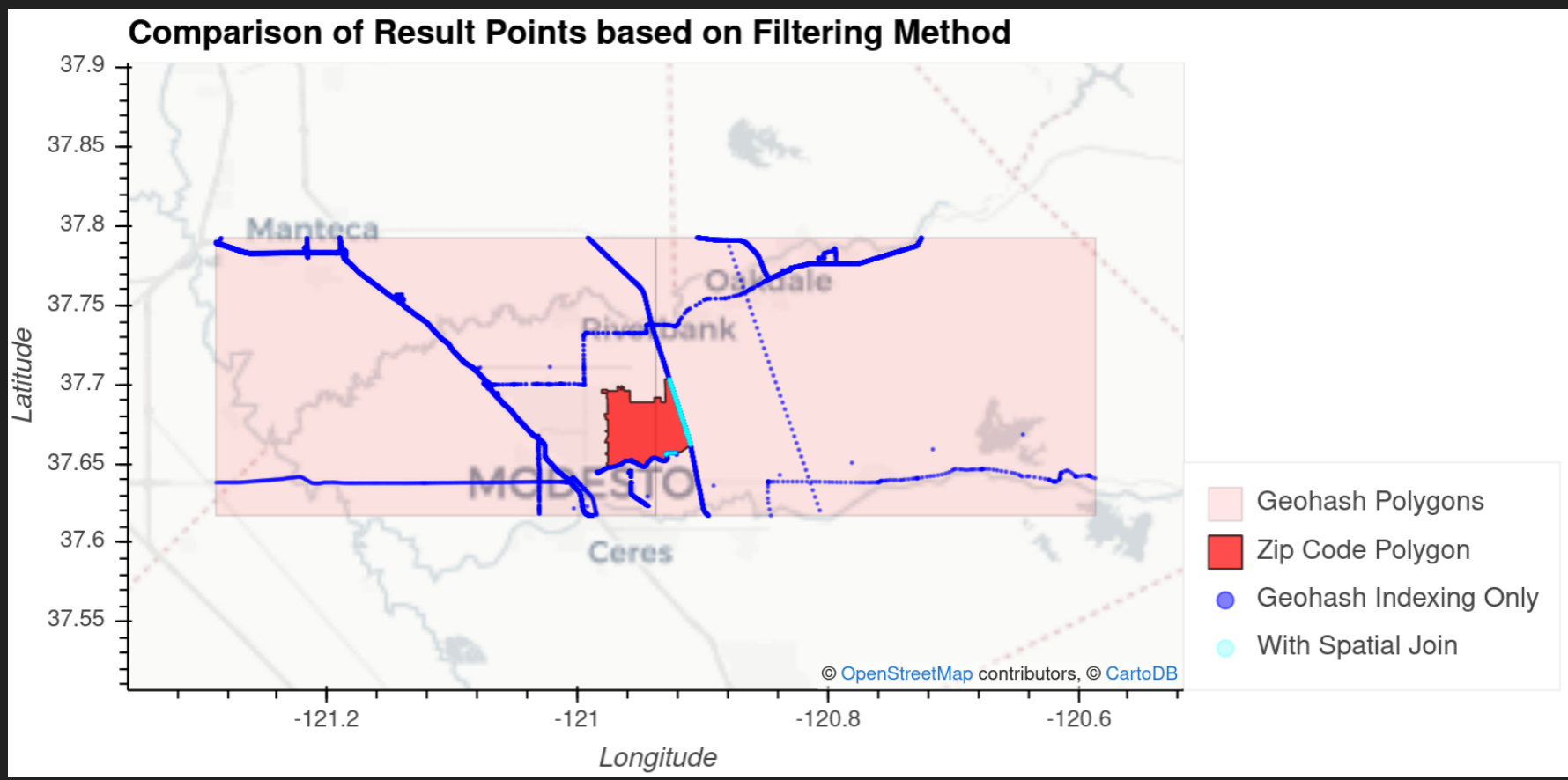

\section{Sorted Geohash Results}

Sorted Geohash w/o spatial join (i.e. no point in polygon filter)

\begin{tabular}{|c|c|c|c|c|c|c|}
\hline \# Polygons & \# Points & $\begin{array}{l}\text { Geohash } \\
\text { Time (min) }\end{array}$ & $\begin{array}{l}\text { Sort Time } \\
\text { (min) }\end{array}$ & $\begin{array}{l}\text { Query Time } \\
\text { (min) }\end{array}$ & $\begin{array}{l}\text { \# Result } \\
\text { Points }\end{array}$ & $\begin{array}{l}\text { Total Time } \\
\text { (min) }\end{array}$ \\
\hline 1 & 113944489 & \multirow{5}{*}{27} & \multirow{5}{*}{3} & 0.05 & 26413 & 29 \\
\hline 10 & 113944489 & & & 0.07 & 156625 & 29 \\
\hline 100 & 113944489 & & & 0.08 & 5269528 & 29 \\
\hline 1000 & 113944489 & & & 0.2 & 38934176 & 29 \\
\hline 10000 & 113944489 & & & 0.4 & 90177639 & 30 \\
\hline
\end{tabular}

Sorted Geohash with spatial join (i.e. with point in polygon filter)

\begin{tabular}{|c|c|c|c|c|c|c|}
\hline \# Polygons & \# Points & $\begin{array}{l}\text { Geohash } \\
\text { Time (min) }\end{array}$ & $\begin{array}{l}\text { Sort Time } \\
\text { (min) }\end{array}$ & $\begin{array}{l}\text { Query Time } \\
\text { (min) }\end{array}$ & $\begin{array}{c}\text { \# Result } \\
\text { Points }\end{array}$ & $\begin{array}{l}\text { Total Time } \\
\text { (min) }\end{array}$ \\
\hline 1 & 113944489 & \multirow{4}{*}{27} & \multirow{4}{*}{3} & 0.07 & 1031 & 29 \\
\hline 10 & 113944489 & & & 0.2 & 6551 & 29 \\
\hline 100 & 113944489 & & & 3 & 203284 & 32 \\
\hline 1000 & 113944489 & & & 27 & 2403824 & 56 \\
\hline
\end{tabular}




\section{Spatialpandas Spatially Sorted}

- Preprocess data if necessary:

- N/A

- Sort data

- Spatially Sort Data using Spatialpandas

- Hilbert Curve

- Spatially Index into Data

- Spatialpandas's sjoin Parallelized with Dask

\section{Spatialpandas Spatially Sorted}

\begin{tabular}{|c|c|c|c|c|c|c|}
\hline \# Polygons & \# Points & $\begin{array}{c}\text { Geohash } \\
\text { Time (s) }\end{array}$ & $\begin{array}{c}\text { Sort Time } \\
\text { (s) }\end{array}$ & $\begin{array}{c}\text { Query Time } \\
\text { (s) }\end{array}$ & $\begin{array}{c}\text { \# Result } \\
\text { Points }\end{array}$ & $\begin{array}{c}\text { Total Time } \\
\text { (s) }\end{array}$ \\
\hline 1 & 113944489 & & & 1 & 1031 & 72 \\
\hline 10 & 113944489 & & & 3 & 6551 & 74 \\
\hline 100 & 113944489 & \multirow{2}{*}{0} & \multirow{2}{*}{71} & 10 & 203284 & 81 \\
\hline 1000 & 113944489 & & & 21 & 2403824 & 92 \\
\hline 10000 & 113944489 & & & 57 & 25877947 & 128 \\
\hline
\end{tabular}




\section{Results}

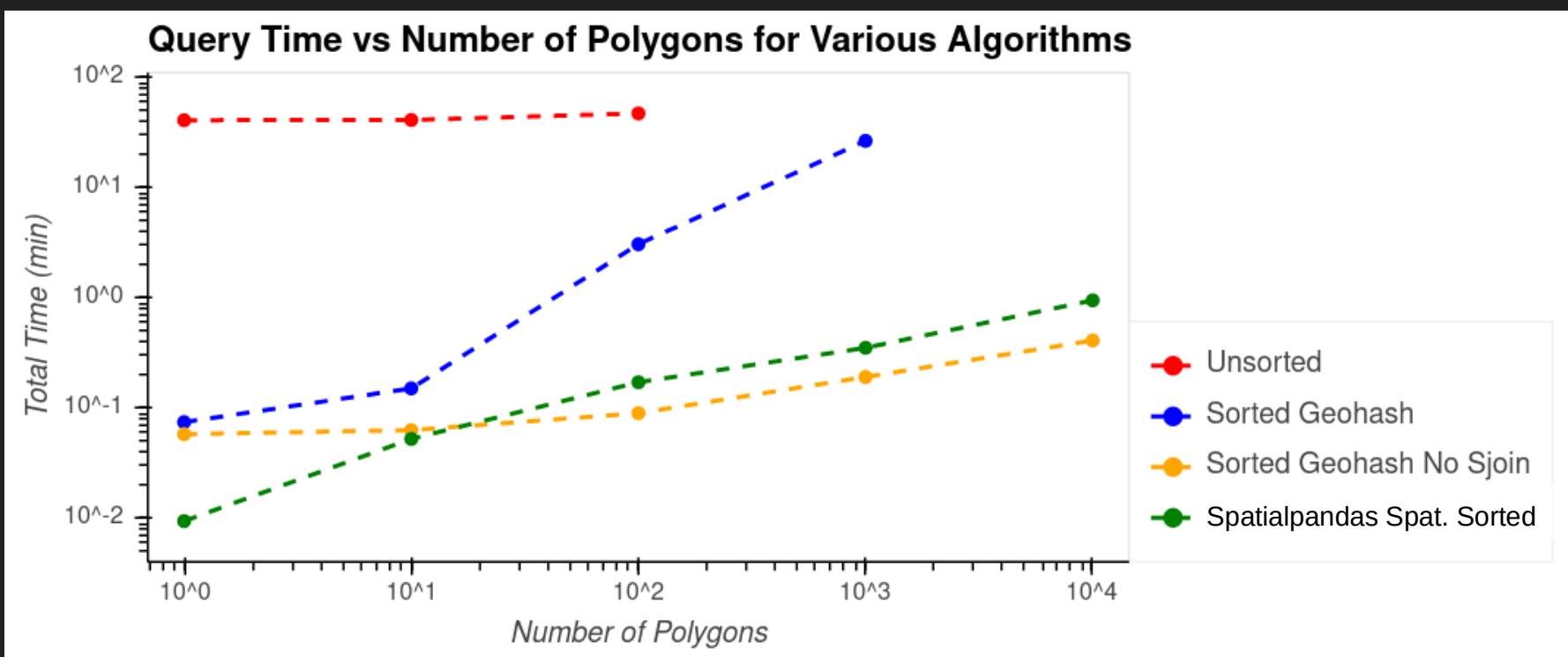

* sorted geohash w/o spatial join is fast but less accurate since it brings in more data than was desired.

\section{Results - One Time Costs}

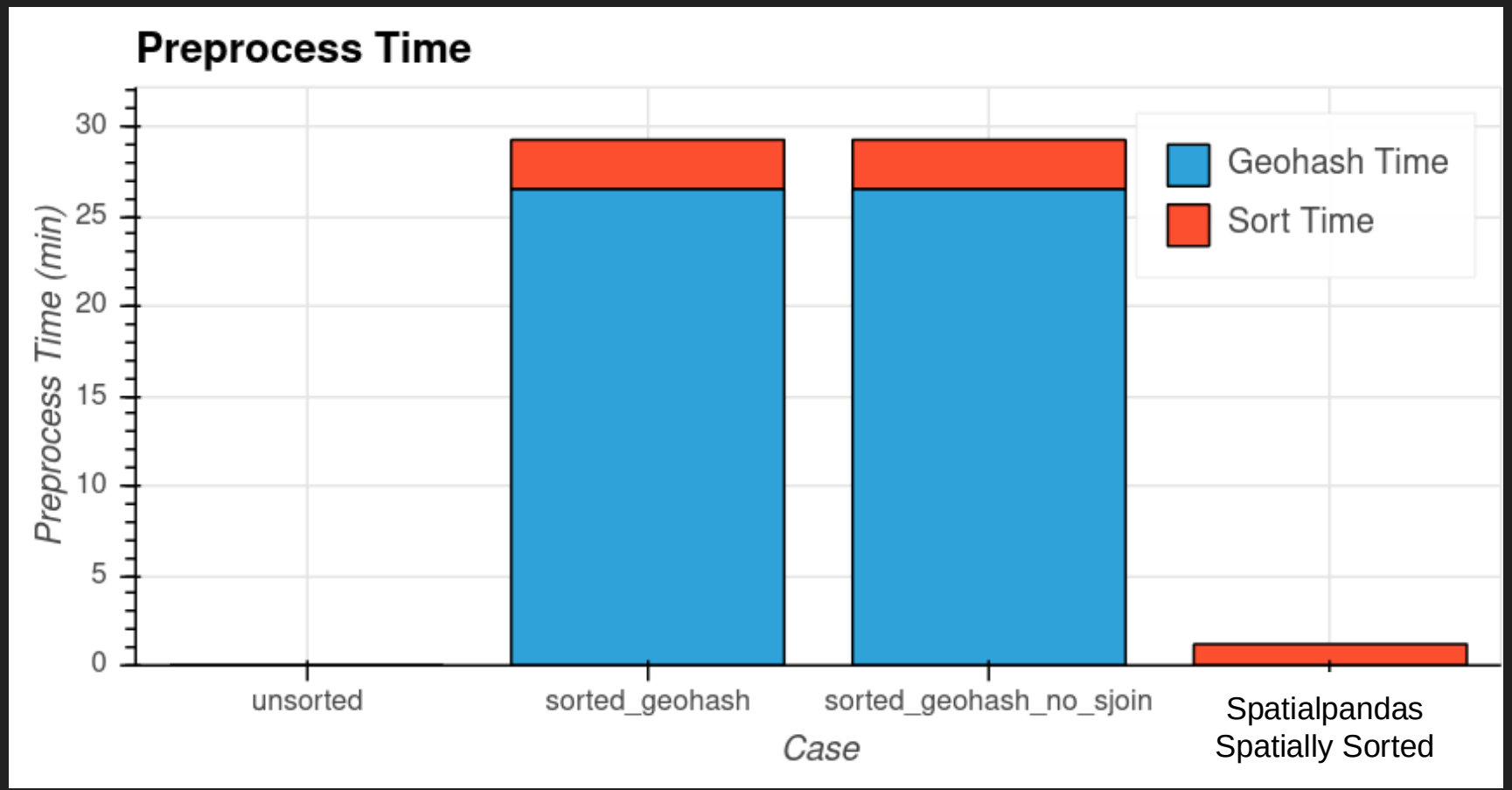




\section{Results Summary}

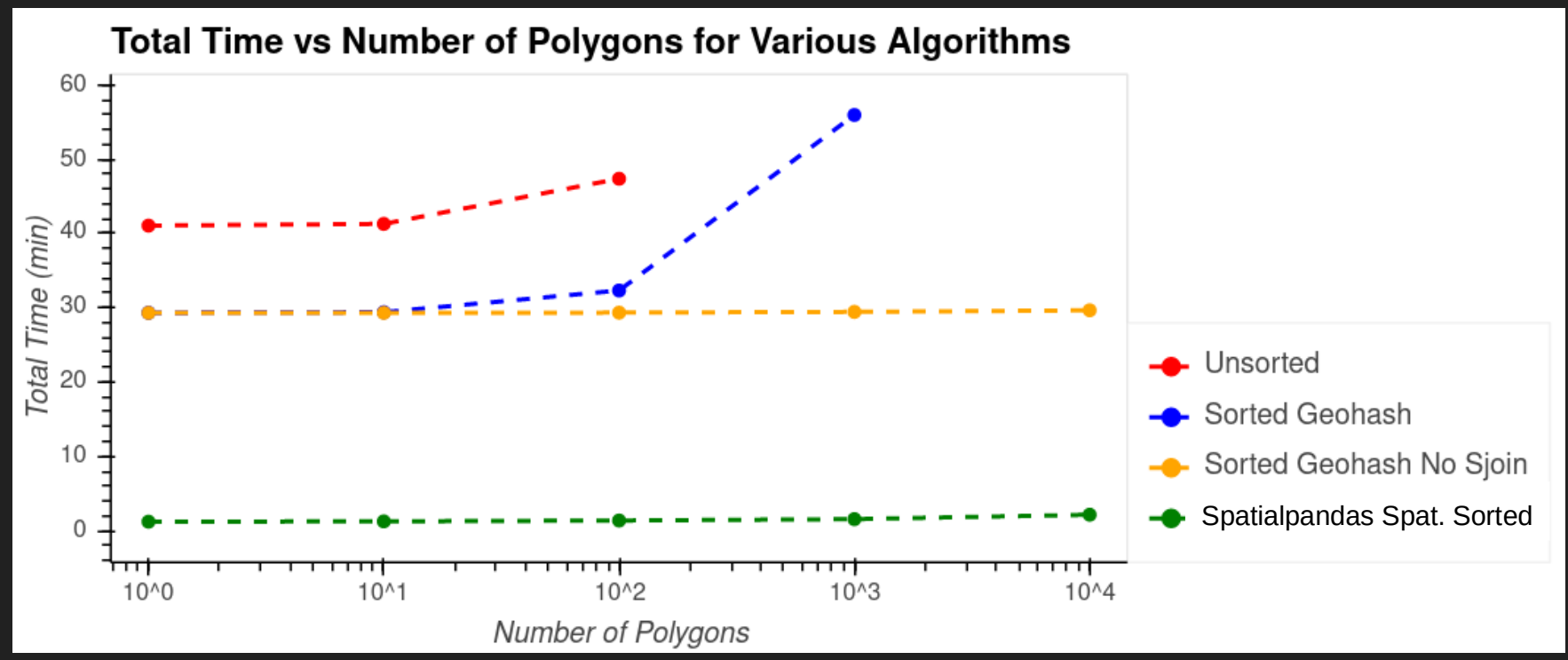

* sorted geohash w/o spatial join is fast but less accurate since it brings in more data than was desired.

\section{SpatialPandas hitps://github.com/holoviz/spatialapandas}

- Advantages:

- Easy to install pure python package

- Vectorized geometry operations implemented with Numba

- Integrated with Dask Dataframe and Parquet

- Contains a vectorized and parallel numba implementation of a Hilbert-RTree.

- Highly efficient parallel spatial joins and bounding box queries on datasets that have been spatially partitioned with spatial pandas

- Disadvantages:

- Young project

- Small community

- Low development activity

Example of spatial sort of country polygons. The images are colored by partition.

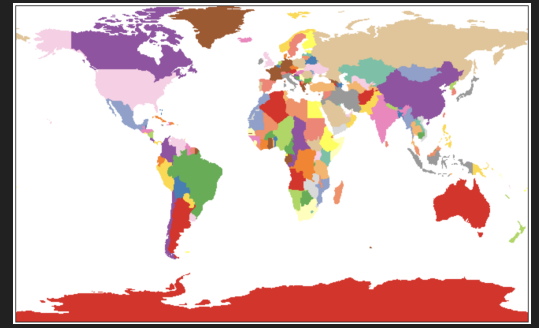

Unsorted Polygons

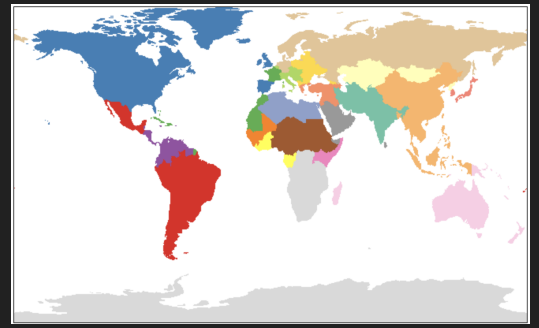

Sorted Polygons 


\section{Thank You!}

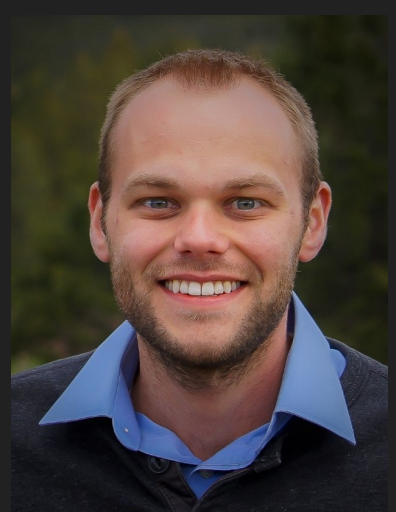

Adam Lewis alewis@quansight.com

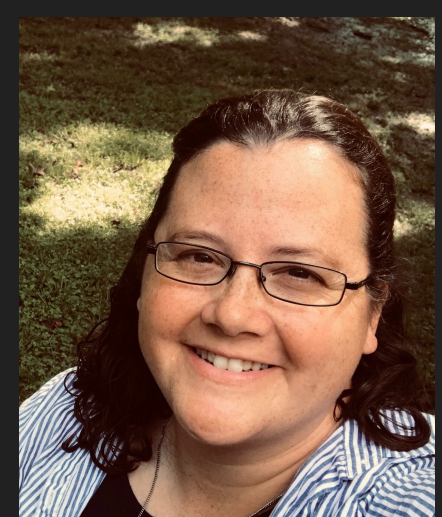

Kim Pevey

kcpevey@quansight.com

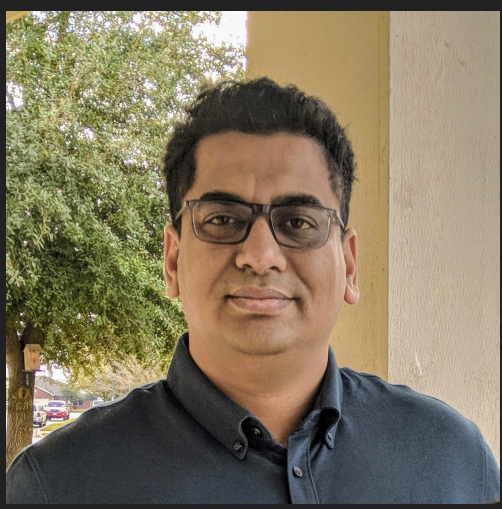

Dharhas Pothina

dharhas@quansight.com

Acknowledgements:

- Jon Mease \& HoloViz Team

- GeoPandas Developers

- Dask Developers

Code and data for benchmarks:

https://github.com/Quansight/scipy2020 spatial algorithms at scale 